\title{
The Almost Sure Local Central Limit Theorem for the Negatively Associated Sequences
}

\author{
Yuanying Jiang ${ }^{1,2}$ and Qunying $W u^{1}$ \\ ${ }^{1}$ College of Science, Guilin University of Technology, Guilin 541004, China \\ ${ }^{2}$ School of Statistics, Renmin University of China, Beijing 100872, China \\ Correspondence should be addressed to Yuanying Jiang; jyy@ruc.edu.cn
}

Received 13 May 2013; Accepted 18 June 2013

Academic Editor: Ying Hu

Copyright (c) 2013 Y. Jiang and Q. Wu. This is an open access article distributed under the Creative Commons Attribution License, which permits unrestricted use, distribution, and reproduction in any medium, provided the original work is properly cited.

In this paper, the almost sure central limit theorem is established for sequences of negatively associated random variables: $\lim _{n \rightarrow \infty}(1 / \log n) \sum_{k=1}^{n}\left(I\left(a_{k} \leq S_{k}<b_{k}\right) / k\right) P\left(a_{k} \leq S_{k}<b_{k}\right)=1$, almost surely. This is the local almost sure central limit theorem for negatively associated sequences similar to results by Csáki et al. (1993). The results extend those on almost sure local central limit theorems from the i.i.d. case to the stationary negatively associated sequences.

\section{Introduction}

Definition 1. Random variables $X_{1}, X_{2}, \ldots, X_{n}, n \geq 2$ are said to be negatively associated (NA) if for every pair of disjoint subsets $A_{1}$ and $A_{2}$ of $\{1,2, \ldots, n\}$,

$$
\operatorname{Cov}\left(f_{1}\left(X_{i}, i \in A_{1}\right), f_{2}\left(X_{j}, j \in A_{2}\right)\right) \leq 0,
$$

where $f_{1}$ and $f_{2}$ are increasing for every variable (or decreasing for every variable) such that this covariance exists. A sequence of random variables $\left\{X_{i}, i \geq 1\right\}$ is said to be NA if every finite subfamily of $\left\{X_{i}, i \geq 1\right\}$ is NA.

Obviously, if $\left\{X_{i}, i \geq 1\right\}$ is a sequence of NA random variables and $\left\{f_{i}, i \geq 1\right\}$ is a sequence of nondecreasing (or nonincreasing) functions, then $\left\{f_{i}\left(X_{i}\right), i \geq 1\right\}$ is also a sequence of NA random variables.

This definition was introduced by the Joag-Dev and Proschan [1]. Statistical test depends greatly on sampling, and the random sampling without replacement from a finite population is NA, but it is not independent. NA sampling has wide applications such as those in multivariate statistical analysis and reliability theory. Because of the wide applications of NA sampling, the notions of NA random variables have received more and more attention recently. We refer to Joag-Dev and Proschan [1] for fundamental properties, Shao
[2] for the moment inequalities, and Wu and Jiang [3] for Chover's law of the iterated logarithm.

Assume that $\left\{X_{n}, n \geq 1\right\}$ is a strictly stationary sequence of NA random variables with $E X_{1}=0,0<E X_{1}^{2}<\infty$. Define $S_{n}=\sum_{j=1}^{n} X_{j}$,

$$
\begin{gathered}
\sigma_{n}^{2}:=\operatorname{Var} S_{n}, \\
\sigma^{2}:=\operatorname{Var} X_{1}+2 \sum_{j=2}^{\infty} \operatorname{Cov}\left(X_{1}, X_{j}\right) .
\end{gathered}
$$

(1) Newman [4] and Matuła [5] showed that NA stationary sequences satisfy the central limit theorem (CLT) under $\sigma^{2}>0$, that is,

$$
\sup _{x \in R}\left|P\left(\frac{S_{n}}{\sigma_{n}}<x\right)-\Phi(x)\right|=o(1) .
$$

(2) Applying Matuła [6] and Wu’s [7] methods, we can easily show that NA sequences satisfy the almost sure central limit theorem (ASCLT), that is,

$$
\lim _{n \rightarrow \infty} \frac{1}{\log n} \sum_{k=1}^{n} \frac{1}{k} I\left\{S_{k} \leq x \sigma k^{1 / 2}\right\}=\Phi(x) \quad \text { a.s. } \forall x \in \mathbb{R},
$$

where $\Phi(x)$ is the standard normal distribution function and $I\{A\}$ denotes the indicator of the event $A$. 
The ASCLT was stimulated by Brosamler [8] and Schatte [9]. Both were concerned with the partial sum of independent and identically distributed (i.i.d.) random variables with more than the second moment. The ASCLT was extensively studied in the past two decades and an interesting direction of the study is to prove it for dependent variables. There are some results for weakly dependent variables such as $\alpha, \rho, \phi$-mixing and associated random variables. Among those results, we refer to Peligrad and Shao [10], Matuła [11], and Wu [7].

More general version of ASCLT was proved by Csáki et al. [12]. The following theorem is due to them.

Theorem A. Let $\left\{X_{n}, n \geq 1\right\}$ be a sequence of i.i.d. random variables with $E\left|X_{1}\right|^{3}<\infty$, let $E X_{1}=0 . a_{k}, b_{k}$ satisfy

$$
-\infty \leq a_{k} \leq 0 \leq b_{k} \leq \infty, \quad k=1,2, \ldots,
$$

and assume that

$$
\sum_{k=1}^{n} \frac{\log k}{k^{3 / 2} P\left(a_{k} \leq S_{k}<b_{k}\right)}=O(\log n), \quad \text { as } n \longrightarrow \infty,
$$

and then

$$
\lim _{n \rightarrow \infty} \frac{1}{\log n} \sum_{k=1}^{n} \frac{I\left\{a_{k} \leq S_{k}<b_{k}\right\}}{k P\left(a_{k} \leq S_{k}<b_{k}\right)}=1 \quad \text { a.s. }
$$

This result may be called almost sure local central limit theorem, while (5) may be called almost sure global central limit theorem. Hurelbaatar [13] extended (8) to the case of $\rho$ mixing sequences and Weng et al. [14] derived an almost sure local central limit theorem for the product of partial sums of a sequence of i.i.d. positive random variables under some regular conditions. For more details, we refer to Berkes and Csáki [15] and Földes [16].

Our concern in this paper is to give a common generalization of (8) to the case of NA sequences.

In the next section we present the exact results, postponing some technical lemmas and the proofs to Section 3.

\section{Main Results}

Assume in the following that $\left\{X_{n}, n \geq 1\right\}$ is a strictly stationary sequence of NA random variables with $E X_{1}=$ $0,0<E X_{1}^{2}<\infty$. We consider the limit behavior of the logarithmic average

$$
\lim _{n \rightarrow \infty} \frac{1}{\log n} \sum_{k=1}^{n} \frac{I\left\{a_{k} \leq S_{k}<b_{k}\right\}}{k P\left(a_{k} \leq S_{k}<b_{k}\right)}
$$

with $-\infty \leq a_{k} \leq 0 \leq b_{k} \leq \infty$, where the terms in the sum above are defined to be 1 if their denominator happens to be 0 .

More precisely let $\left\{a_{n}, n \geq 1\right\}$ and $\left\{b_{n}, n \geq 1\right\}$ be two sequences of real numbers and put

$$
\begin{gathered}
p_{k}:=P\left(a_{k} \leq S_{k}<b_{k}\right), \\
\alpha_{k}:= \begin{cases}\frac{I\left\{a_{k} \leq S_{k}<b_{k}\right\}}{p_{k}}, & \text { if } p_{k} \neq 0, \\
1, & \text { if } p_{k}=0 .\end{cases}
\end{gathered}
$$

So we need to investigate the limit behavior of

$$
\mu_{n}:=\sum_{k=1}^{n} \frac{\alpha_{k}}{k}
$$

under certain conditions.

In our considerations, we will need the following CoxGrimmett coefficient which describes the covariance structure of the sequence

$$
u(n):=\sup _{k \in N} \sum_{j:|j-k| \geq n}\left|\operatorname{Cov}\left(X_{j}, X_{k}\right)\right|, \quad n \in N \cup\{0\} .
$$

We remark that for a stationary sequence of NA random variables

$$
u(n)=-2 \sum_{k=n+1}^{\infty} \operatorname{Cov}\left(X_{1}, X_{k}\right), \quad n \in N
$$

By Lemma 8 of Newman [4], we have $u(0)<\infty$ and $\lim _{n \rightarrow \infty} u(n)=0$.

In the following, $\xi_{n} \sim \eta_{n}$ denotes $\xi_{n} / \eta_{n} \rightarrow 1, n \rightarrow \infty$. $\xi_{n}=O\left(\eta_{n}\right)$ denotes that there exists a constant $c>0$ such that $\xi_{n} \leq c \eta_{n}$ for sufficiently large $n$. The symbols $c, c_{1}, c_{2}, \ldots$, stand for generic positive constants which may differ from one place to another.

Theorem 2. Let $\left\{X_{n}, n \geq 1\right\}$ be a strictly stationary sequence of $N A$ random variables with $E X_{1}=0, E\left|X_{1}\right|^{3}<\infty$ and let $\sigma^{2}>0 . a_{k}, b_{k}$ satisfy (6). Assume that

$$
\sum_{n=1}^{\infty} u(n)<\infty
$$

and for some $\beta>1$,

$$
\sum_{\substack{1 \leq k \leq n \\ p_{k} \neq 0}} \frac{(\log k)^{1 / 3}}{k p_{k}}=O\left((\log n)^{2}(\log \log n)^{-\beta}\right) .
$$

Then we have

$$
\lim _{n \rightarrow \infty} \frac{\mu_{n}}{\log n}=1, \quad \text { a.s., }
$$

where $\mu_{n}$ is defined by (11).

Remark 3. Let $a_{k}=-\infty$ and $b_{k}=x \sigma k^{1 / 2}$ in (6). By the central limit theorem (4), we have $p_{k}=P\left(S_{k} / \sigma k^{1 / 2}<x\right) \rightarrow \Phi(x)$, obviously (15) satisfies; then (16) becomes (5), which is the almost sure global central limit theorem. Thus the almost sure local central limit theorem is a general result which contains the almost sure global central limit theorem.

Remark 4. The condition (15) is satisfied with a wide range of $p_{k}$; for example, if

$$
p_{k}=0 \quad \text { or } \quad p_{k} \geq c \frac{(\log \log k)^{\beta}}{(\log k)^{2 / 3}}
$$


holds, then the condition (15) is satisfied. In fact, letting $0<$ $\delta<1$, we have

$$
\begin{aligned}
\sum_{\substack{1 \leq k \leq n \\
p_{k} \neq 0}} & \frac{(\log k)^{1 / 3}}{k p_{k}} \\
& \leq c \sum_{1 \leq k \leq n}(\log \log k)^{-\beta}(\log k)^{\delta} \frac{(\log k)^{1-\delta}}{k} \\
& \leq c(\log \log n)^{-\beta}(\log n)^{\delta} \sum_{1 \leq k \leq n} \frac{(\log k)^{1-\delta}}{k} \\
& \leq c(\log \log n)^{-\beta}(\log n)^{2} \\
& =O\left((\log n)^{2}(\log \log n)^{-\beta}\right) .
\end{aligned}
$$

In the given theorem below, we strengthen the condition (6) on $a_{k}$ and $b_{k}$. Meanwhile, as a compensation, we do not need to impose restricting condition (15) on $p_{k}$.

Theorem 5. Let $\left\{X_{n}, n \geq 1\right\}$ be a strictly stationary sequence of $N A$ random variables with $E X_{1}=0, E\left|X_{1}\right|^{3}<\infty$, and $\sigma^{2}>0$, and let $a_{k}$ and $b_{k}$ satisfy

$$
\begin{gathered}
-c_{1} k^{1 / 2-\alpha} \leq a_{k} \leq-c_{2} k^{1 / 2-\alpha}, \\
c_{3} k^{1 / 2-\alpha} \leq b_{k} \leq c_{4} k^{1 / 2-\alpha},
\end{gathered}
$$

where $0<\alpha<1 / 7$. Assume that (14) hold, and then we have (16).

\section{Proofs}

The following lemmas play important roles in the proof of our theorems. The proofs are given in the Appendix.

Lemma 6. Assume that $\left\{\xi_{n}, n \geq 1\right\}$ are random variables such that

$$
\xi_{k} \geq 0, \quad E \xi_{k}=1, \quad k=1,2, \ldots
$$

and furthermore there exists $d_{k} \geq 0$ such that $D_{n}=\sum_{k=1}^{n} d_{k} \uparrow$ $\infty, D_{n} / D_{n-1} \rightarrow 1$, and

$$
\operatorname{Var}\left(\sum_{k=1}^{n} d_{k} \xi_{k}\right) \leq c D_{n}^{2}\left(\log D_{n}\right)^{-\beta},
$$

with some $\beta>1$ and positive constant $c$, and then

$$
\lim _{n \rightarrow \infty} \frac{1}{D_{n}} \sum_{k=1}^{n} d_{k} \xi_{k}=1 \quad \text { a.s. }
$$

Remark 7. Let $d_{k}=1 / k$ in (21), and then $D_{n}=\sum_{k=1}^{n} 1 / k \sim$ $\log n$. Thus, if

$$
\operatorname{Var}\left(\sum_{k=1}^{n} \frac{1}{k} \xi_{k}\right) \leq c(\log n)^{2}(\log \log n)^{-\beta},
$$

with some $\beta>1$ and positive constant $c$, then

$$
\lim _{n \rightarrow \infty} \frac{1}{\log n} \sum_{k=1}^{n} \frac{1}{k} \xi_{k}=1 \quad \text { a.s. }
$$

The following Lemma 8 is obvious.

Lemma 8. Assume that the nonnegative random sequence $\left\{\xi_{n}, n \geq 1\right\}$ satisfies (24) and the sequence $\left\{\eta_{n}, n \geq 1\right\}$ is such that, for any $\varepsilon>0$, there exists a $k_{0}=k_{0}(\varepsilon, \omega)$ for which

$$
(1-\varepsilon) \xi_{k} \leq \eta_{k} \leq(1+\varepsilon) \xi_{k}, \quad k>k_{0} .
$$

Then we have also

$$
\lim _{n \rightarrow \infty} \frac{1}{\log n} \sum_{k=1}^{n} \frac{1}{k} \eta_{k}=1 \quad \text { a.s. }
$$

The following Lemma 9 is an easy corollary to the Corollary 2.2 in Matuła [5] under strictly stationary condition, which studies the rate of convergence in the CLT under negative dependence. It was also studied in Pan [17]. Of course this is the Berry-Esseen inequality for the NA sequence.

Lemma 9. Let $\left\{X_{j}, j \in N\right\}$ be a strictly stationary sequence of $N A$ random variables with $E X_{1}=0, E\left|X_{1}\right|^{3}<\infty, \sigma^{2}>0$ satisfying (14). Then one has

$$
\sup _{x \in R}\left|P\left(\frac{S_{n}}{\sigma_{n}}<x\right)-\Phi(x)\right|=O\left(n^{-1 / 5}\right) .
$$

Lemma 10. If the conditions of Lemma 9 hold, $a_{k}$ and $b_{k}$ satisfy (19). Then one has

$$
c_{1}^{\prime} k^{-\alpha} \leq p_{k} \leq c_{2}^{\prime} k^{-\alpha} \quad\left(k \geq k_{0}\right)
$$

where $\alpha$ is as (19).

Lemma 11. If the conditions of Lemma 9 hold, $a_{k}$ and $b_{k}$ satisfy (19), and $\alpha$ is as (19). Assume that $\varepsilon_{l}=l^{3 \alpha / 2}$, and then the following asymptotic relations hold:

$$
\begin{gathered}
\sum_{\substack{1 \leq k<l \leq n \\
k<l-l^{\alpha}}} \frac{1}{k l p_{l}} P\left(\left|S_{l^{\alpha}}\right| \geq \varepsilon_{l}\right)=O(\log n), \\
\sum_{\substack{1 \leq k<l \leq n \\
k<l-l^{\alpha}}} \frac{1}{k l p_{l}} \frac{1}{\left(l-k-l^{\alpha}\right)^{1 / 5}}=O(\log n), \\
\sum_{\substack{1 \leq k<l \leq n \\
k<l-l^{\alpha}}} \frac{1}{k l p_{l}}\left|\Phi\left(\frac{a_{l}-b_{k}-\varepsilon_{l}}{\left(l-k-l^{\alpha}\right)^{1 / 2}}\right)-\Phi\left(\frac{a_{l}}{l^{1 / 2}}\right)\right| \\
=O(\log n), \\
\sum_{\substack{1 \leq k<l \leq n \\
k<l-l^{\alpha}}} \frac{1}{k l p_{l}}\left|\left(\Phi\left(\frac{b_{l}-a_{k}+\varepsilon_{l}}{\left(l-k-l^{\alpha}\right)^{1 / 2}}\right)-\Phi\left(\frac{b_{l}}{l^{1 / 2}}\right)\right)\right| \\
=O(\log n) .
\end{gathered}
$$


The main point in our proof is to verify the condition (23). We use global central limit theorem with remainders and the following elementary inequalities:

$$
|\Phi(x)-\Phi(y)| \leq c|x-y|, \quad \text { for every } x, y \in \mathbb{R}
$$

with some constant $c$. Moreover, for each $k>0$, there exists $c_{1}=c_{1}(k)$, such that

$$
\begin{aligned}
& |\Phi(x)-\Phi(y)| \\
& \quad \geq c_{1}|x-y|, \quad \text { for every } x, y \in \mathbb{R},|x|+|y| \leq k .
\end{aligned}
$$

Let $\left\{X_{n}, n \geq 1\right\}$ be a strictly stationary sequence of NA random variables with $\sigma^{2}>0$; we can immediately get $\sigma_{n}^{2} \sim$ $n \sigma^{2}$, that is,

$$
c_{1} n \leq \operatorname{Var}\left(S_{n}\right)=\sigma_{n}^{2} \leq c_{2} n
$$

for some constant $c_{1}, c_{2}>0$ and sufficiently large $n$.

Proof of Theorem 2. First assume that

$$
b_{k}-a_{k} \leq c k^{1 / 2}, \quad k=1,2, \ldots
$$

with some constant $c$. Let $1 \leq k<l$ and $\varepsilon_{k}=k^{1 / 2}(\log k)^{1 / 3}$.

If either $p_{k}=0$ or $p_{l}=0$, then obviously $\operatorname{Cov}\left(\alpha_{k}, \alpha_{l}\right)=0$, and so we may assume that $p_{k} p_{l} \neq 0$; then, we have

$$
\begin{aligned}
& \operatorname{Cov}\left(\alpha_{k}, \alpha_{l}\right) \\
& =\frac{1}{p_{l} p_{k}}\left(P\left(a_{k} \leq S_{k}<b_{k}, a_{l} \leq S_{l}<b_{l}\right)-p_{l} p_{k}\right) \\
& \leq \frac{1}{p_{l} p_{k}}\left(P \left(a_{l}-b_{k} \leq S_{l}-S_{k}<b_{l}-a_{k},\right.\right. \\
& \left.\left.\quad a_{k} \leq S_{k}<b_{k}\right)-p_{l} p_{k}\right) \\
& \leq \frac{1}{p_{l} p_{k}}\left(P\left(a_{l}-b_{k} \leq S_{l}-S_{k}<b_{l}-a_{k}\right)\right. \\
& \left.\quad \times P\left(a_{k} \leq S_{k}<b_{k}\right)-p_{l} p_{k}\right) \\
& =\frac{1}{p_{l}}\left(P\left(a_{l}-b_{k} \leq S_{l}-S_{k}<b_{l}-a_{k}\right)-p_{l}\right) \\
& \leq \frac{1}{p_{l}}\left(P\left(a_{l}-b_{k}-\varepsilon_{k}<S_{l}<b_{l}-a_{k}+\varepsilon_{k}\right)\right. \\
& \left.\quad-p_{l}+P\left(\left|S_{k}\right| \geq \varepsilon_{k}\right)\right) \\
& \leq \frac{1}{p_{l}}\left(P\left(a_{l}-b_{k}-\varepsilon_{k} \leq S_{l}<a_{l}\right)\right. \\
& \left.\quad+P\left(b_{l} \leq S_{l}<b_{l}-a_{k}+\varepsilon_{k}\right)+P\left(\left|S_{k}\right| \geq \varepsilon_{k}\right)\right) .
\end{aligned}
$$

Applying Lemma 9, (33), (35), and (36) and noting that $\varepsilon_{k}=k^{1 / 2}(\log k)^{1 / 3}$, we obtain

$$
\begin{aligned}
P( & \left.a_{l}-b_{k}-\varepsilon_{k} \leq S_{l}<a_{l}\right)+P\left(b_{l} \leq S_{l}<b_{l}-a_{k}+\varepsilon_{k}\right) \\
\leq & \left(\Phi\left(\frac{a_{l}}{\sigma_{l}}\right)-\Phi\left(\frac{a_{l}-b_{k}-\varepsilon_{k}}{\sigma_{l}}\right)\right) \\
& +\left(\Phi\left(\frac{b_{l}-a_{k}+\varepsilon_{k}}{\sigma_{l}}\right)-\Phi\left(\frac{b_{l}}{\sigma_{l}}\right)\right)+c \frac{1}{l^{1 / 5}} \\
& \leq \frac{b_{k}+\varepsilon_{k}}{\sigma_{l}}+\frac{-a_{k}+\varepsilon_{k}}{\sigma_{l}}+c \frac{1}{l^{1 / 5}}=\frac{b_{k}-a_{k}}{\sigma_{l}}+\frac{2 \varepsilon_{k}}{\sigma_{l}}+c \frac{1}{l^{1 / 5}} \\
\leq & c\left(\frac{k^{1 / 2}}{l^{1 / 2}}+\frac{\varepsilon_{k}}{l^{1 / 2}}+\frac{1}{l^{1 / 5}}\right) \leq c\left(\frac{k^{1 / 2}(\log k)^{1 / 3}}{l^{1 / 2}}+\frac{1}{l^{1 / 5}}\right) .
\end{aligned}
$$

By the condition of (15), we have

$$
\begin{aligned}
& \sum_{l=1}^{n} \sum_{k=1}^{l} \frac{1}{k l p_{l}} \frac{k^{1 / 2}(\log k)^{1 / 3}}{l^{1 / 2}} \\
& \leq \sum_{l=1}^{n} \frac{1}{l^{3 / 2} p_{l}} \sum_{k=1}^{l} \frac{(\log k)^{1 / 3}}{k^{1 / 2}} \\
& \leq c \sum_{l=1}^{n} \frac{(\log l)^{1 / 3}}{l^{3 / 2} p_{l}} \sum_{k=1}^{l} \frac{1}{k^{1 / 2}} \leq c \sum_{l=1}^{n} \frac{(\log l)^{1 / 3}}{l^{3 / 2} p_{l}} l^{1 / 2} \\
& \leq c \sum_{l=1}^{n} \frac{(\log l)^{1 / 3}}{l p_{l}}=O\left((\log n)^{2}(\log \log n)^{-\beta}\right),
\end{aligned}
$$

$$
\begin{aligned}
& \sum_{l=1}^{n} \sum_{k=1}^{l-1} \frac{1}{k l p_{l}} \frac{1}{l^{1 / 5}} \\
& \quad=\sum_{l=1}^{n} \frac{1}{l^{6 / 5} p_{l}} \sum_{k=1}^{l-1} \frac{1}{k} \leq c \sum_{l=1}^{n} \frac{1}{l^{6 / 5} p_{l}} \log l \\
& \leq c \sum_{l=1}^{n} \frac{(\log l)^{1 / 3}}{l p_{l}}=O\left((\log n)^{2}(\log \log n)^{-\beta}\right) .
\end{aligned}
$$

By Chebyshev's inequality and the condition of (15) and (35), we obtain

$$
\begin{aligned}
& \sum_{l=1}^{n} \sum_{k=1}^{l} \frac{1}{k l p_{l}} P\left(\left|S_{k}\right| \geq \varepsilon_{k}\right) \\
& \quad \leq \sum_{l=1}^{n} \frac{1}{l p_{l}} \sum_{k=1}^{l} \frac{\operatorname{Var}\left(S_{k}\right)}{k \varepsilon_{k}^{2}} \leq c \sum_{l=1}^{n} \frac{1}{l p_{l}} \sum_{k=1}^{l} \frac{1}{k(\log k)^{2 / 3}} \\
& \quad \leq c \sum_{l=1}^{n} \frac{(\log l)^{1 / 3}}{l p_{l}}=O\left((\log n)^{2}(\log \log n)^{-\beta}\right) .
\end{aligned}
$$

Hence (37)-(40) imply that

$$
\sum_{l=1}^{n} \sum_{k=1}^{l-1} \frac{\operatorname{Cov}\left(\alpha_{k}, \alpha_{l}\right)}{k l}=O\left((\log n)^{2}(\log \log n)^{-\beta}\right) .
$$


But $\operatorname{Var}\left(\alpha_{k}\right)=0$ if $p_{k}=0$ and

$$
\operatorname{Var}\left(\alpha_{k}\right)=\frac{1-p_{k}}{p_{k}} \leq \frac{1}{p_{k}} \quad \text { if } p_{k} \neq 0
$$

Thus

$$
\begin{aligned}
& \sum_{k=1}^{n} \frac{\operatorname{Var}\left(\alpha_{k}\right)}{k^{2}} \\
& \quad \leq \sum_{\substack{1 \leq k \leq n \\
p_{k} \neq 0}} \frac{1}{k^{2} p_{k}} \leq \sum_{\substack{1 \leq k \leq n \\
p_{k} \neq 0}} \frac{(\log k)^{1 / 3}}{k p_{k}} \\
& \quad=O\left((\log n)^{2}(\log \log n)^{-\beta}\right) .
\end{aligned}
$$

Equations (41) and (43) together imply that

$$
\operatorname{Var}\left(\sum_{k=1}^{n} \frac{\alpha_{k}}{k}\right)=O\left((\log n)^{2}(\log \log n)^{-\beta}\right), \quad \text { as } n \rightarrow \infty \text {. }
$$

Hence applying Remark 7, our theorem is proved under the restricting condition (36). define

Now we drop the restricting condition (36). Fix $x>0$ and

$$
\begin{gathered}
\tilde{a}_{k}=\max \left(a_{k},-x \sigma k^{1 / 2}\right), \\
\tilde{b}_{k}=\min \left(b_{k}, x \sigma k^{1 / 2}\right), \\
\tilde{p}_{k}=P\left(\widetilde{a}_{k} \leq S_{k}<\widetilde{b}_{k}\right),
\end{gathered}
$$

where $\sigma$ is defined by (3).

Clearly $\widetilde{p}_{k} \leq p_{k}$, and so assuming $\widetilde{p}_{k} \neq 0$; then, also we have $p_{k} \neq 0$, and thus

$$
\begin{aligned}
\alpha_{k}= & \frac{1}{p_{k}} I\left\{a_{k} \leq S_{k}<b_{k}\right\} \\
= & \frac{1}{p_{k}}\left(I\left\{\tilde{a}_{k} \leq S_{k}<\widetilde{b}_{k}\right\}\right. \\
& \left.\quad+I\left\{a_{k} \leq S_{k}<\tilde{a}_{k}\right\}+I\left\{\widetilde{b}_{k} \leq S_{k}<b_{k}\right\}\right) \\
\leq & \frac{1}{\widetilde{p}_{k}} I\left\{\tilde{a}_{k} \leq S_{k}<\widetilde{b}_{k}\right\} \\
& +\frac{1}{p_{k}}\left(I\left\{a_{k} \leq S_{k}<\widetilde{a}_{k}\right\}+I\left\{\widetilde{b}_{k} \leq S_{k}<b_{k}\right\}\right) \\
\leq & \frac{1}{\widetilde{p}_{k}} I\left\{\widetilde{a}_{k} \leq S_{k}<\widetilde{b}_{k}\right\} \\
& +\frac{I\left\{S_{k}<-x \sigma k^{1 / 2}\right\}}{P\left(-x \sigma k^{1 / 2} \leq S_{k}<0\right)}+\frac{I\left\{S_{k} \geq x \sigma k^{1 / 2}\right\}}{P\left(0 \leq S_{k}<x \sigma k^{1 / 2}\right)} .
\end{aligned}
$$

By (35) and the central limit theorem for NA random variables (4), that is,

$$
\sup _{x \in R}\left|P\left(\frac{S_{n}}{\sigma_{n}}<x\right)-\Phi(x)\right|=o(1),
$$

we obtain

$$
\begin{aligned}
& \lim _{k \rightarrow \infty} P\left(-x \sigma k^{1 / 2} \leq S_{k}<0\right) \\
& \quad=\lim _{k \rightarrow \infty} P\left(-x \sigma_{k} \leq S_{k}<0\right)=\Phi(0)-\Phi(-x), \\
& \lim _{k \rightarrow \infty} P\left(0 \leq S_{k}<x \sigma k^{1 / 2}\right) \\
& \quad=\lim _{k \rightarrow \infty} P\left(0 \leq S_{k}<x \sigma_{k}\right)=\Phi(x)-\Phi(0) .
\end{aligned}
$$

Applying the almost sure central limit theorem for NA random variables (5), that is,

$$
\lim _{n \rightarrow \infty} \frac{1}{\log n} \sum_{k=1}^{n} \frac{1}{k} I\left\{S_{k} \leq x \sigma k^{1 / 2}\right\}=\Phi(x) \quad \text { a.s. } \forall x \in \mathbb{R},
$$

Lemma 8, and (48), we have

$$
\begin{aligned}
\lim _{n \rightarrow \infty} & \frac{1}{\log n} \sum_{k=1}^{n} \frac{I\left\{S_{k}<-x \sigma k^{1 / 2}\right\}}{k P\left(-x \sigma k^{1 / 2} \leq S_{k}<0\right)} \\
& =\frac{\Phi(-x)}{\Phi(0)-\Phi(-x)} \quad \text { a.s., } \\
\lim _{n \rightarrow \infty} & \frac{1}{\log n} \sum_{k=1}^{n} \frac{I\left\{S_{k}>x \sigma k^{1 / 2}\right\}}{k P\left(0 \leq S_{k}<x \sigma k^{1 / 2}\right)} \\
= & \frac{1-\Phi(x)}{\Phi(x)-\Phi(0)} \quad \text { a.s. }
\end{aligned}
$$

Since $\tilde{a}_{k}$ and $\tilde{b}_{k}$ satisfy (36), we get

$$
\lim _{n \rightarrow \infty} \frac{1}{\log n} \sum_{k=1}^{n} \frac{\tilde{\alpha}_{k}}{k}=1 \quad \text { a.s., }
$$

where

$$
\tilde{\alpha}_{k}= \begin{cases}\frac{I\left\{\tilde{a}_{k} \leq S_{k}<\tilde{b}_{k}\right\}}{\tilde{p}_{k}}, & \text { if } \tilde{p}_{k} \neq 0, \\ 1, & \text { if } \tilde{p}_{k}=0 .\end{cases}
$$

Equations (46) and (50)-(51) together imply that

$$
\limsup _{n \rightarrow \infty} \frac{1}{\log n} \sum_{k=1}^{n} \frac{\alpha_{k}}{k} \leq 1+2 \frac{1-\Phi(x)}{\Phi(x)-\Phi(0)} \text { a.s. }
$$

On the other hand, if $\widetilde{p}_{k} \neq 0$, then we have

$$
\begin{aligned}
& \frac{1}{p_{k}} I\left\{a_{k} \leq S_{k}<b_{k}\right\} \\
& \geq \frac{1}{\widetilde{p}_{k}} I\left\{\widetilde{a}_{k} \leq S_{k}<\widetilde{b}_{k}\right\}\left(1-\frac{p_{k}-\tilde{p}_{k}}{p_{k}}\right) \\
& \geq \widetilde{\alpha}_{k}\left(1-\frac{P\left(S_{k}<-\sigma x k^{1 / 2}\right)+P\left(S_{k}>\sigma x k^{1 / 2}\right)}{\min \left\{P\left(0 \leq S_{k}<\sigma x k^{1 / 2}\right), P\left(-\sigma x k^{1 / 2} \leq S_{k}<0\right)\right\}}\right),
\end{aligned}
$$


and by the central limit theorem,

$$
\begin{aligned}
\lim _{k \rightarrow \infty} & \frac{P\left(S_{k}<-\sigma x k^{1 / 2}\right)+P\left(S_{k}>\sigma x k^{1 / 2}\right)}{\min \left\{P\left(0 \leq S_{k}<\sigma x k^{1 / 2}\right), P\left(-\sigma x k^{1 / 2} \leq S_{k}<0\right)\right\}} \\
& =1-2 \frac{1-\Phi(x)}{\Phi(x)-\Phi(0)} .
\end{aligned}
$$

Applying Lemma 8, (51) and (54) imply that

$$
\liminf _{n \rightarrow \infty} \frac{1}{\log n} \sum_{k=1}^{n} \frac{\alpha_{k}}{k} \geq 1-2 \frac{1-\Phi(x)}{\Phi(x)-\Phi(0)} \quad \text { a.s., }
$$

and hence

$$
\begin{aligned}
1+2 \frac{1-\Phi(x)}{\Phi(x)-\Phi(0)} & \geq \limsup _{n \rightarrow \infty} \frac{1}{\log n} \sum_{k=1}^{n} \frac{\alpha_{k}}{k} \\
& \geq \liminf _{n \rightarrow \infty} \frac{1}{\log n} \sum_{k=1}^{n} \frac{\alpha_{k}}{k} \\
& \geq 1-2 \frac{1-\Phi(x)}{\Phi(x)-\Phi(0)} \text { a.s. }
\end{aligned}
$$

By the arbitrariness of $x$, let $x \rightarrow \infty$ in (57); we have

$$
\begin{aligned}
1 & \geq \limsup _{n \rightarrow \infty} \frac{1}{\log n} \sum_{k=1}^{n} \frac{\alpha_{k}}{k} \\
& \geq \liminf _{n \rightarrow \infty} \frac{1}{\log n} \sum_{k=1}^{n} \frac{\alpha_{k}}{k} \geq 1 \quad \text { a.s. }
\end{aligned}
$$

Thus

$$
\lim _{n \rightarrow \infty} \frac{1}{\log n} \sum_{k=1}^{n} \frac{\alpha_{k}}{k}=1 \text { a.s. }
$$

This completes the proof of Theorem 2.

Proof of Theorem 5. Let $k<l-l^{\alpha}, 1 \leq k<l$, and $\varepsilon_{l}=l^{3 \alpha / 2}$; we have

$$
\begin{aligned}
& \operatorname{Cov}\left(\alpha_{k}, \alpha_{l}\right) \\
& \leq \frac{1}{p_{l} p_{k}}\left(P \left(a_{l}-b_{k} \leq S_{l}-S_{k+l^{\alpha}}+S_{k+l^{\alpha}}-S_{k}\right.\right. \\
& \left.\left.\quad<b_{l}-a_{k}, a_{k} \leq S_{k}<b_{k}\right)-p_{l} p_{k}\right) \\
& \leq \frac{1}{p_{l} p_{k}}\left(P \left(a_{l}-b_{k} \leq S_{l}-S_{k+l^{\alpha}}+S_{k+l^{\alpha}}-S_{k}\right.\right. \\
& \left.\left.\quad<b_{l}-a_{k}\right) P\left(a_{k} \leq S_{k}<b_{k}\right)-p_{l} p_{k}\right) \\
& \leq \frac{1}{p_{l}}\left(P\left(a_{l}-b_{k}-\varepsilon_{l}<S_{l}-S_{k+l^{\alpha}}<b_{l}-a_{k}+\varepsilon_{l}\right)\right. \\
& \left.-p_{l}+P\left(\left|S_{k+l^{\alpha}}-S_{k}\right| \geq \varepsilon_{l}\right)\right) \\
& \leq \frac{1}{p_{l}}\left(P\left(a_{l}-b_{k}-\varepsilon_{l}<S_{l-k-l^{\alpha}}<b_{l}-a_{k}+\varepsilon_{l}\right)\right. \\
& \left.-p_{l}+P\left(\left|S_{l^{\alpha}}\right| \geq \varepsilon_{l}\right)\right) .
\end{aligned}
$$

Applying Lemma 9, (33), and (35), we obtain

$$
\begin{gathered}
P\left(a_{l}-b_{k}-\varepsilon_{l}<S_{l-k-l^{\alpha}}<b_{l}-a_{k}+\varepsilon_{l}\right)-p_{l} \\
\leq\left(\Phi\left(\frac{b_{l}-a_{k}+\varepsilon_{l}}{\left(l-k-l^{\alpha}\right)^{1 / 2}}\right)-\Phi\left(\frac{b_{l}}{l^{1 / 2}}\right)\right) \\
+\left(\Phi\left(\frac{a_{l}}{l^{1 / 2}}\right)-\Phi\left(\frac{a_{l}-b_{k}-\varepsilon_{l}}{\left(l-k-l^{\alpha}\right)^{1 / 2}}\right)\right) \\
+c\left(\frac{1}{l^{1 / 5}}+\frac{1}{\left(l-k-l^{\alpha}\right)^{1 / 5}}\right) .
\end{gathered}
$$

Hence Lemma 11, (60), and (61) imply that

$$
\sum_{\substack{1 \leq k<l \leq n \\ k<l-l^{\alpha}}} \frac{\operatorname{Cov}\left(\alpha_{k}, \alpha_{l}\right)}{k l}=O(\log n) .
$$

On the other hand,

$$
\sum_{\substack{1 \leq k<l \leq n \\ k>l-l^{\alpha}}} \frac{\operatorname{Cov}\left(\alpha_{k}, \alpha_{l}\right)}{k l}=O(\log n)
$$

because $l-l^{\alpha}<k<l, l^{\gamma}-\left(l-l^{\alpha}\right)^{\gamma} \rightarrow 0$ as $l \rightarrow \infty$ for $\gamma<1, \alpha<1$.

$$
\begin{aligned}
& \text { But } \operatorname{Var}\left(\alpha_{k}\right)=0 \text { if } p_{k}=0 \text { and } \\
& \qquad \operatorname{Var}\left(\alpha_{k}\right)=\frac{1-p_{k}}{p_{k}} \leq \frac{1}{p_{k}} \text { if } p_{k} \neq 0 .
\end{aligned}
$$

Then

$$
\sum_{k=1}^{n} \frac{\operatorname{Var}\left(\alpha_{k}\right)}{k^{2}} \leq \sum_{\substack{1 \leq k \leq n \\ p_{k} \neq 0}} \frac{1}{k^{2} p_{k}} \leq \sum_{k=1}^{n} \frac{1}{k^{5 / 2-\alpha}}=O(\log n) .
$$

Noting that

$$
\begin{aligned}
\operatorname{Var}\left(\sum_{k=1}^{n} \frac{\alpha_{k}}{k}\right) \\
=\sum_{k=1}^{n} \frac{\operatorname{Var}\left(\alpha_{k}\right)}{k^{2}} \\
\quad+2 \sum_{\substack{1 \leq k<l \leq n \\
k>l-l^{\alpha}}} \frac{\operatorname{Cov}\left(\alpha_{k}, \alpha_{l}\right)}{k l}+2 \sum_{\substack{1 \leq k<l \leq n \\
k<l-l^{\alpha}}} \frac{\operatorname{Cov}\left(\alpha_{k}, \alpha_{l}\right)}{k l},
\end{aligned}
$$

thus (62)-(66) imply that

$$
\operatorname{Var}\left(\sum_{k=1}^{n} \frac{\alpha_{k}}{k}\right)=O(\log n), \quad \text { as } n \longrightarrow \infty .
$$

Hence applying Remark 7, we have

$$
\lim _{n \rightarrow \infty} \frac{1}{\log n} \sum_{k=1}^{n} \frac{\alpha_{k}}{k}=1 \text { a.s. }
$$

This completes the proof of Theorem 5 . 


\section{Appendix}

Proof of Lemma 6. Let $\mu_{n}=\sum_{k=1}^{n} d_{k} \xi_{k}$, and then $\forall \varepsilon>0$

$$
P\left(\left|\frac{\mu_{n}}{D_{n}}-\frac{E \mu_{n}}{D_{n}}\right| \geq \varepsilon\right) \leq \frac{\operatorname{Var}\left(\mu_{n} / D_{n}\right)}{\varepsilon^{2}} \leq c\left(\log D_{n}\right)^{-\beta} .
$$

Let $r<1, r \beta>1$, and $n_{k}=\inf \left\{n_{k}, D_{n_{k}} \geq \exp \left(k^{r}\right)\right\}$, and then $D_{n_{k}} \geq \exp \left(k^{r}\right), D_{n_{k}-1}<\exp \left(k^{r}\right)$, for $D_{n} \sim D_{n-1}$; we get

$$
1 \leq \frac{D_{n_{k}}}{\exp \left(k^{r}\right)} \sim \frac{D_{n_{k}-1}}{\exp \left(k^{r}\right)}<1 \longrightarrow 1
$$

that is,

$$
D_{n_{k}} \sim \exp \left(k^{r}\right)
$$

and thus

$$
\begin{aligned}
\frac{D_{n_{k}}}{D_{n_{k-1}}} & \sim \frac{\exp \left(k^{r}\right)}{\exp \left((k-1)^{r}\right)} \\
& =\exp \left(k^{r}\left[1-\left(1-\frac{1}{k}\right)^{r}\right]\right) \sim \exp \left(k^{r} \cdot r \cdot \frac{1}{k}\right) \\
& =\exp \left(r \cdot k^{r-1}\right) .
\end{aligned}
$$

On account of $r<1$, then

$$
\begin{gathered}
\frac{D_{n_{k}}}{D_{n_{k-1}}} \sim \exp \left(r \cdot k^{r-1}\right) \longrightarrow 1, \quad \text { as } k \rightarrow \infty, \\
\sum_{k=1}^{\infty} P\left(\left|\frac{\mu_{n_{k}}}{D_{n_{k}}}-\frac{E \mu_{n_{k}}}{D_{n_{k}}}\right| \geq \varepsilon\right) \\
\quad \leq c \sum_{k=1}^{\infty} \frac{1}{\left(\log D_{n}\right)^{-\beta}} \leq c \sum_{k=1}^{\infty} \frac{1}{k^{r \beta}}<\infty .
\end{gathered}
$$

By the Borel-Cantelli lemma,

Since

$$
\frac{\mu_{n_{k}}}{D_{n_{k}}}-\frac{E \mu_{n_{k}}}{D_{n_{k}}} \longrightarrow 0 \text { a.s. }
$$

thus

$$
\frac{E \mu_{n_{k}}}{D_{n_{k}}}=\frac{\sum_{k=1}^{n_{k}} d_{k}}{D_{n_{k}}}=1,
$$

$$
\frac{\mu_{n_{k}}}{D_{n_{k}}} \longrightarrow 1, \quad \text { a.s. }
$$

Now for $n_{k-1} \leq n<n_{k}$, for $D_{n} \uparrow \infty, D_{n_{k}} / D_{n_{k-1}} \rightarrow 1$, and by $\xi_{k} \geq 0$, then $\mu_{n} \uparrow$, and we have

$$
1 \longleftarrow \frac{D_{n_{k}-1}}{D_{n_{k}}} \frac{\mu_{n_{k-1}}}{D_{n_{k-1}}} \leq \frac{\mu_{n}}{D_{n}} \leq \frac{\mu_{n_{k}}}{D_{n_{k}}} \frac{D_{n_{k}}}{D_{n_{k-1}}} \longrightarrow 1 \quad \text { a.s. }
$$

hence

$$
\frac{\mu_{n}}{D_{n}} \longrightarrow 1 \text { a.s. }
$$

This completes the proof of Lemma 6.
Proof of Lemma 10. Applying Lemma 9, (33), and noting the conditions of (19) and $0<\alpha<1 / 7$, we get

$$
\begin{aligned}
p_{k} & =P\left(a_{k} \leq S_{k} \leq b_{k}\right) \\
& \leq\left(\Phi\left(\frac{b_{k}}{k^{1 / 2}}\right)-\Phi\left(\frac{a_{k}}{k^{1 / 2}}\right)\right)+c \frac{1}{k^{1 / 5}} \\
& \leq c\left(\frac{k^{1 / 2-\alpha}}{k^{1 / 2}}+\frac{1}{k^{1 / 5}}\right) \leq c_{2}^{\prime} k^{-\alpha} .
\end{aligned}
$$

Applying Lemma 9, (34), and noting the conditions of (19) and $0<\alpha<1 / 7$, we have

$$
\begin{aligned}
p_{k} & =P\left(a_{k} \leq S_{k} \leq b_{k}\right) \\
& \geq\left(\Phi\left(\frac{b_{k}}{k^{1 / 2}}\right)-\Phi\left(\frac{a_{k}}{k^{1 / 2}}\right)\right)-c \frac{1}{k^{1 / 5}} \\
& \geq c\left(\frac{k^{1 / 2-\alpha}}{k^{1 / 2}}+\frac{1}{k^{1 / 5}}\right) \geq c_{1}^{\prime} k^{-\alpha} .
\end{aligned}
$$

Thus Lemma 10 immediately follows from (A.11) and (A.12).

Proof of Lemma 11. By Lemma 10, Chebyshev's inequality, and noting the condition of $0<\alpha<1 / 7$, we have

$$
\begin{aligned}
& \sum_{\substack{1 \leq k<l \leq n \\
k<l-l^{\alpha}}} \frac{1}{k l p_{l}} P\left(\left|S_{l^{\alpha}}\right| \geq \varepsilon_{l}\right) \\
& \quad \leq \sum_{\substack{1 \leq k<l \leq n \\
k<l-l^{\alpha}}} \frac{1}{k l p_{l}} \frac{\operatorname{Var}\left(S_{l^{\alpha}}\right)}{l^{3 \alpha}} \leq c \sum_{l=1}^{n} \frac{1}{l^{1+\alpha}} \sum_{k=1}^{l-l^{\alpha}} \frac{1}{k} \\
& \leq c \sum_{l=1}^{n} \frac{\log \left(l-l^{\alpha}\right)}{l^{1+\alpha}}=O(\log n) .
\end{aligned}
$$

It proves (29). By Lemma 10 and $0<\alpha<1 / 7$, we get

$$
\begin{aligned}
& \sum_{\substack{1 \leq k<l \leq n \\
k<l-l^{\alpha}}} \frac{1}{k l p_{l}} \frac{1}{\left(l-k-l^{\alpha}\right)^{1 / 5}} \\
& \quad \leq c \sum_{l=1}^{n} \frac{1}{l^{1-\alpha}}\left(\sum_{l \leq k<\left(l-l^{\alpha}\right) / 2} \frac{1}{k\left(l-l^{\alpha}-k\right)^{1 / 5}}\right.
\end{aligned}
$$$$
\left.+\sum_{\left(l-l^{\alpha}\right) / 2<k<l-l^{\alpha}} \frac{1}{k\left(l-l^{\alpha}-k\right)^{1 / 5}}\right)
$$$$
\leq c \sum_{l=1}^{n} \frac{1}{l^{1-\alpha}}\left(\frac{1}{\left(l-l^{\alpha}\right)^{1 / 5}} \sum_{1 \leq k<\left(l-l^{\alpha}\right) / 2} \frac{1}{k}\right.
$$$$
\left.+\frac{1}{l-l^{\alpha}} \sum_{1 \leq k<\left(l-l^{\alpha}\right) / 2} \frac{1}{k^{1 / 5}}\right)
$$

$$
\leq c \sum_{l=1}^{n} \frac{\log l}{l^{1-\alpha+1 / 5}} \leq c \sum_{l=1}^{n} \frac{1}{l}=O(\log n) .
$$


It proves (30). Applying Lemma 9, (33), (35), $\varepsilon_{l}=l^{3 \alpha / 2}$, and noting the condition of $0<\alpha<1 / 7$, we obtain

$$
\begin{aligned}
& \sum_{\substack{1 \leq k<l \leq n \\
k<l-l^{\alpha}}} \frac{1}{k l p_{l}}\left|\Phi\left(\frac{a_{l}-b_{k}-\varepsilon_{l}}{\left(l-k-l^{\alpha}\right)^{1 / 2}}\right)-\Phi\left(\frac{a_{l}}{l^{1 / 2}}\right)\right| \\
& \leq \underset{\substack{1 \leq k<l \leq n \\
k<l-l^{\alpha}}}{\leq} \frac{-a_{l}}{k l p_{l}}\left(\frac{1}{\left(l-k-l^{\alpha}\right)^{1 / 2}}-\frac{1}{\sqrt{l}}\right) \\
& +\underset{\substack{1 \leq k<l \leq n \\
k<l-l^{\alpha}}}{c} \frac{1}{k l p_{l}} \frac{b_{k}}{\left(l-k-l^{\alpha}\right)^{1 / 2}} \\
& +c \sum_{\substack{1 \leq k<l \leq n \\
k<l-l^{\alpha}}} \frac{1}{k l p_{l}} \frac{\varepsilon_{l}}{\left(l-k-l^{\alpha}\right)^{1 / 2}}:=\Sigma_{1}+\Sigma_{2}+\Sigma_{3} .
\end{aligned}
$$

Now applying the same procedure as before, we have

$$
\begin{aligned}
& \Sigma_{1} \leq c \sum_{\substack{1 \leq k<l \leq n \\
k<l-l^{\alpha}}} \frac{-a_{l}}{k l p_{l}}\left(\frac{1}{l^{1-\alpha}\left(l-k-l^{\alpha}\right)^{1 / 2}}+\frac{k}{l\left(l-k-l^{\alpha}\right)^{1 / 2}}\right) \\
& \leq c \sum_{1 \leq l \leq n} \frac{1}{l^{3 / 2-\alpha}} \\
& \times\left(\frac{1}{\left(1-l^{\alpha}\right)^{1 / 2}} \sum_{1 \leq k<\left(l-l^{\alpha}\right) / 2} \frac{1}{k}+\frac{1}{l-l^{\alpha}} \sum_{1 \leq k<\left(l-l^{\alpha}\right) / 2} \frac{1}{k^{1 / 2}}\right) \\
& +c \sum_{1 \leq l \leq n} \frac{1}{l^{3 / 2}} \sum_{1 \leq k<l-l^{\alpha}} \frac{1}{\left(l-k-l^{\alpha}\right)^{1 / 2}} \\
& \leq c \sum_{1 \leq l \leq n} \frac{\log l}{l^{2-\alpha}} \leq c \sum_{1 \leq l \leq n} \frac{1}{l}=O(\log n) \\
& \Sigma_{2} \leq c \sum_{\substack{1 \leq k<l \leq n \\
k<l-l^{\alpha}}} \frac{1}{l^{1-\alpha} k^{1 / 2+\alpha}\left(l-l^{\alpha}-k\right)^{1 / 2}} \\
& \leq c \sum_{1 \leq l \leq n} \frac{1}{l^{1-\alpha}}\left(\frac{1}{\left(l-l^{\alpha}\right)^{1 / 2}} \sum_{1 \leq k<\left(l-l^{\alpha}\right) / 2} \frac{1}{k^{1 / 2+\alpha}}\right. \\
& \left.+\frac{1}{\left(l-l^{\alpha}\right)^{1 / 2+\alpha}} \sum_{1 \leq k<\left(l-l^{\alpha}\right) / 2} \frac{1}{k^{1 / 2}}\right) \\
& \leq c \sum_{1 \leq l \leq n} \frac{1}{l^{1-\alpha}}\left(\frac{1}{\left(l-l^{\alpha}\right)^{1 / 2}} l^{1 / 2-\alpha}+\frac{1}{\left(l-l^{\alpha}\right)^{1 / 2+\alpha}}\left(l-l^{\alpha}\right)^{1 / 2}\right) \\
& \leq c \sum_{1 \leq l \leq n} \frac{1}{l}=O(\log n) \text {. }
\end{aligned}
$$

Noting that $0<\alpha<1 / 7$, we deduce

$$
\begin{aligned}
\Sigma_{3} \leq & c \sum_{\substack{1 \leq k<l \leq n \\
k<l-l^{\alpha}}} \frac{1}{l^{1-(7 / 2) \alpha} k^{1 / 2}\left(l-l^{\alpha}-k\right)^{1 / 2}} \\
\leq & c \sum_{1 \leq l \leq n} \frac{1}{l^{1-(7 / 2) \alpha}} \\
& \times\left(\frac{1}{\left(l-l^{\alpha}\right)^{1 / 2}} \sum_{1 \leq k<\left(l-l^{\alpha}\right) / 2} \frac{1}{k}+\frac{1}{\left(l-l^{\alpha}\right)} \sum_{1 \leq k<\left(l-l^{\alpha}\right) / 2} \frac{1}{k^{1 / 2}}\right) \\
\leq & c \sum_{1 \leq l \leq n} \frac{\log l}{l^{3 / 2-(7 / 2) \alpha}} \leq c \sum_{1 \leq l \leq n} \frac{1}{l}=O(\log n) .
\end{aligned}
$$

It proves (31). The proof of (32) is similar to the proof of (31). This completes the proof of Lemma 11.

\section{Acknowledgments}

The authors are very grateful to the academic editor, professor Ying $\mathrm{Hu}$, and the two anonymous reviewers for their valuable comments and helpful suggestions, which significantly contributed to improving the quality of this paper. This work is jointly supported by National Natural Science Foundation of China (11061012,71271210), Project Supported by Program to Sponsor Teams for Innovation in the Construction of Talent Highlands in Guangxi Institutions of Higher Learning ((2011)47), the Guangxi China Science Foundation (2013GXNSFDA019001).

\section{References}

[1] K. Joag-Dev and F. Proschan, "Negative association of random variables, with applications," The Annals of Statistics, vol. 11, no. 1, pp. 286-295, 1983.

[2] Q. M. Shao, "A comparison theorem on moment inequalities between negatively associated and independent random variables," Journal of Theoretical Probability, vol. 13, no. 2, pp. 343356, 2000.

[3] Q. Y. Wu and Y. Y. Jiang, "A law of the iterated logarithm of partial sums for NA random variables," Journal of the Korean Statistical Society, vol. 39, no. 2, pp. 199-206, 2010.

[4] C. M. Newman, "Asymptotic independence and limit theorems for positively and negatively dependent random variables," in Inequalities in Statistics and Probability, vol. 5 of Lecture Notes-Monograph Series, pp. 127-140, Institute of Mathematical Statistics, Hayward, Calif, USA, 1984.

[5] P. Matuła, "Some limit theorems for negatively dependent random variables," Yokohama Mathematical Journal, vol. 41, no. 2, pp. 163-173, 1994.

[6] P. Matuła, "On almost sure limit theorems for positively dependent random variables," Statistics \& Probability Letters, vol. 74, no. 1, pp. 59-66, 2005.

[7] Q. Y. Wu, "An almost sure central limit theorem for the weight function sequences of NA random variables," ProceedingsMathematical Sciences, vol. 121, no. 3, pp. 369-377, 2011.

[8] G. A. Brosamler, "An almost everywhere central limit theorem," Mathematical Proceedings of the Cambridge Philosophical Society, vol. 104, no. 3, pp. 561-574, 1988. 
[9] P. Schatte, "On strong versions of the central limit theorem," Mathematische Nachrichten, vol. 137, pp. 249-256, 1988.

[10] M. Peligrad and Q. M. Shao, "A note on the almost sure central limit theorem for weakly dependent random variables," Statistics \& Probability Letters, vol. 22, no. 2, pp. 131-136, 1995.

[11] P. Matuła, "On the almost sure central limit theorem for associated random variables," Probability and Mathematical Statistics, vol. 18, no. 2, pp. 411-416, 1998.

[12] E. Csáki, A. Földes, and P. Révész, "On almost sure local and global central limit theorems," Probability Theory and Related Fields, vol. 97, no. 3, pp. 321-337, 1993.

[13] G. Hurelbaatar, "An almost sure local and global central limit theorem for weakly dependent random variables," Annales Universitatis Scientiarum Budapestinensis de Rolando Eötvös Nominatae, Sectio Mathematica, vol. 38, pp. 109-126, 1995.

[14] Z. C. Weng, Z. C. Peng, and S. Nadarajah, "The almost sure local central limit theorem for the product of partial sums," Proceedings-Mathematical Sciences, vol. 121, no. 2, pp. 217-228, 2011.

[15] I. Berkes and E. Csáki, "A universal result in almost sure central limit theory," Stochastic Processes and their Applications, vol. 94, no. 1, pp. 105-134, 2001.

[16] A. Földes, "Some of my favorite results with Endre Csáki and Pál Révész: a survey, Periodica Mathematica Hungarica, vol. 50, no. 1-2, pp. 117-134, 2005.

[17] J. M. Pan, "On the convergence rate in the central limit theorem for negatively associated sequences," Chinese Journal of Applied Probability and Statistics, vol. 13, no. 2, pp. 183-192, 1997. 


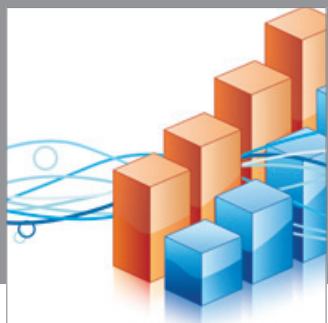

Advances in

Operations Research

mansans

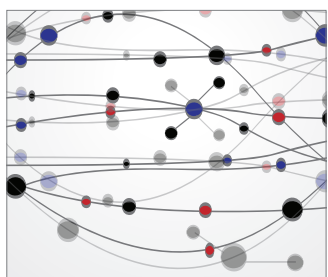

The Scientific World Journal
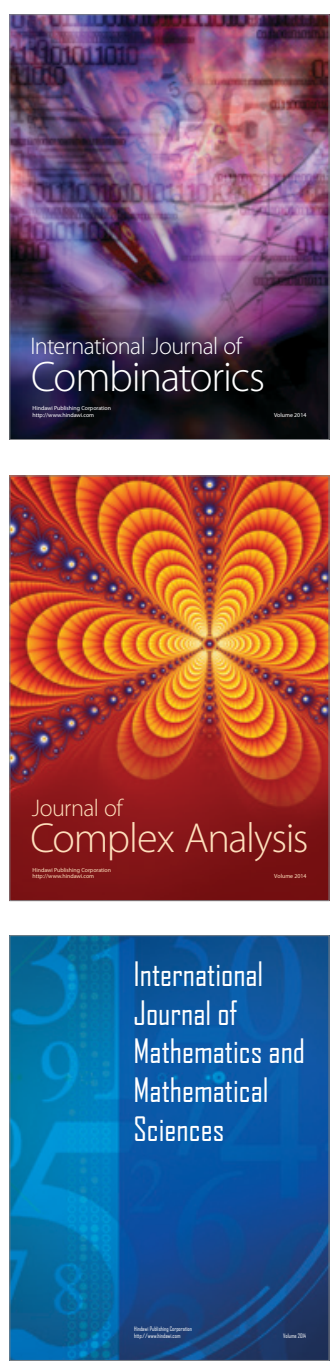
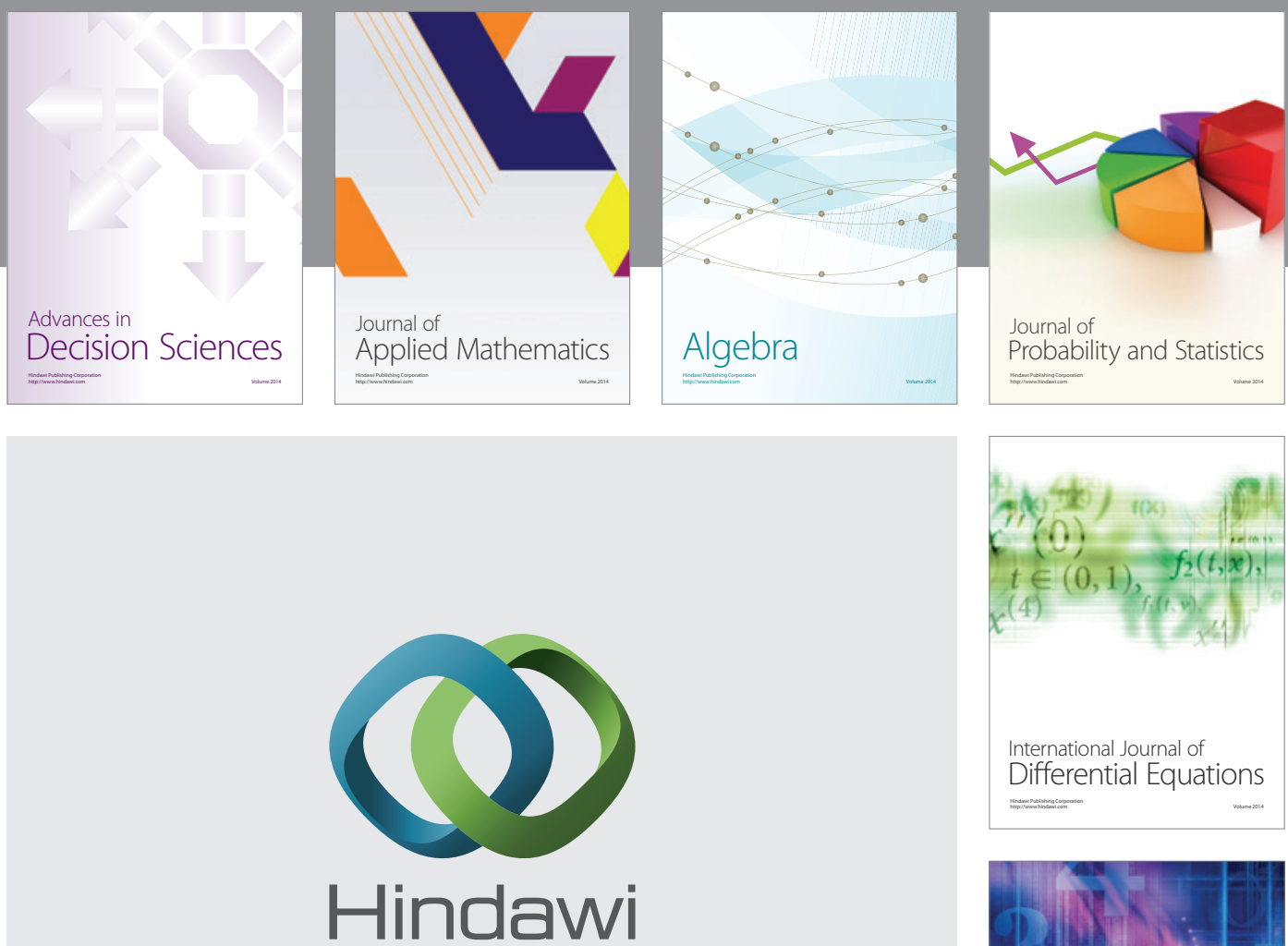

Submit your manuscripts at http://www.hindawi.com
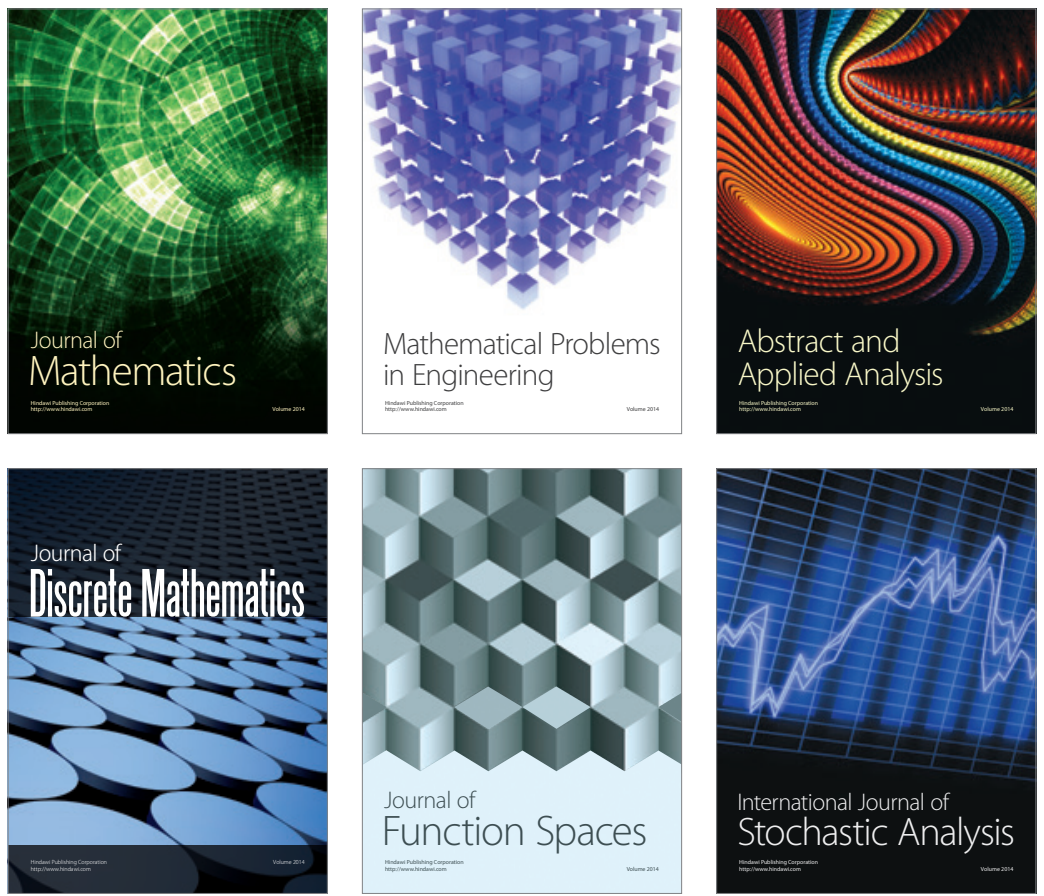

Journal of

Function Spaces

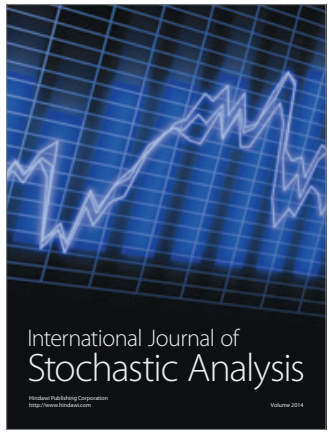

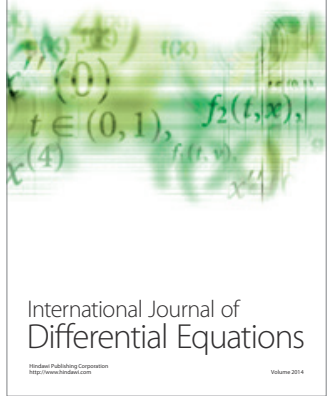
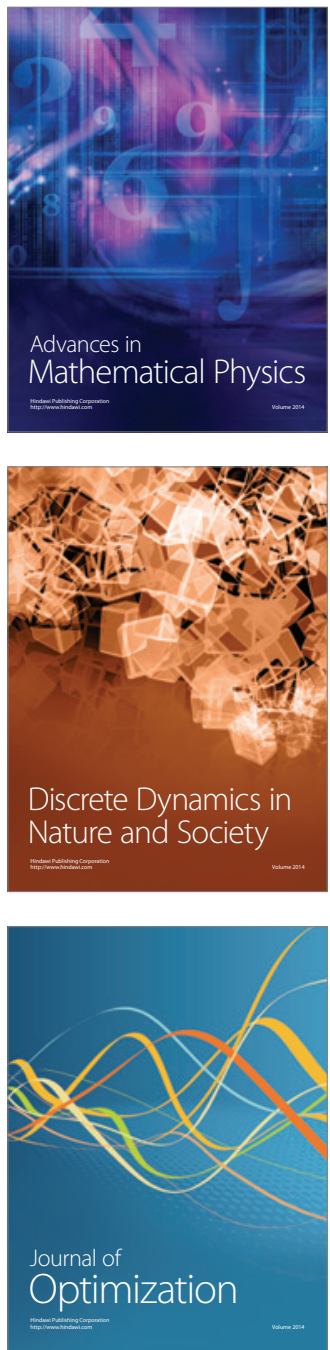